\title{
miRNAGE-34 induces cardiac damAGE
}

Cell Research (2013) 23:866-867. doi:10.1038/cr.2013.57; published online 23 April 2013

\section{Small non-coding microRNAs} (miRNAs, miRs) regulate gene expression in virtually all cells, and they have been implicated in cardiovascular disease and aging. In a paper recently published in Nature, miR-34a was identified as an agingassociated apoptotic and overall damaging factor for the heart.

The miRNA canonical but not exclusive mechanism of action involves the targeting of messenger RNAs (mRNAs) by either complementary or semi-complementary binding between a short $(8$ bp) miRNA "seed sequence" and one or more miRNA binding regions in the mRNA 3' untranslated region (UTR), thus inhibiting gene expression. An individual miRNA directly targets a plethora of genes and multiple miRNAs can compete for the same mRNA targets, which creates intricate regulatory networks able to fine-tuning molecular pathways affecting biological and pathophysiological functions, including cell death, lifespan and aging.

In a recent paper in Nature, Boon and colleagues elegantly show upregulation of miR-34a in the heart during aging in humans and mice [1]. Corroborating evidences are derived from work on mice with loss of Ku80. The Ku80 unit has been implicated in multiple processes, including DNA repair, telomere maintenance and apoptosis. Ku80 knockout mice are more prone to cancer and age prematurely [2]. Interestingly, miR-34a level is increased in the heart of Ku 80 heterozygous and homozygous knockout mice. In the latter, inhibition of miR-34a expression by systemic use of a miRNA inhibitor retarded agedependent cardiac deterioration [1].
The other members of miR-34 (b and c) were also overexpressed in the aged heart. However, they are produced as a bicistronic unit from a different chromosome than miR-34a and regulation of this miRNA family is not entirely clear.

Boon et al. showed that age-associated miR-34a upregulation is more robust than that of miR-34b-c. In the heart, miR-34a localizes mainly in cardiomyocytes, but also in other cardiac cell types where its function is less clear. Cardiac aging is associated with increased apoptosis, fibrosis deposition, hypertrophy and reduced function. All these features could be corrected by systemic use of miR-34a inhibitors [1] (Figure 1). Whether this miRNA is more upstream than others in aging processes remains to be determined as other miRNAs involved in cardiac aging have been identified [3].

miR-34 level is elevated in the mouse infarcted heart $[1,4]$, where its inhibition proved therapeutically useful by acting on the aforementioned processes and additionally promoting post-ischemic reparative angiogenesis (Figure 1). Similar beneficial effects were evident using a miR-34a knockout model. The myocardial infarct (MI) data by Boon et al. [1] are partially in line with Bernardo et al. [5], which proved the therapeutic benefit of the full miR-34 family inhibition in both mouse MI and pressure overload via transverse aortic constriction (TAC) models. However, they did not find miR-34a inhibition alone able to gain a therapeutic benefit [5]. The appeal of miRNA-34a as a therapeutic target is apparent and deserves further translational efforts to tackle ischemic heart disease, especially that associated with aging. It remains to be elucidated whether miR-34a inhibition would produce an even stronger therapeutic effect when MI is induced in spontaneously or prematurely aged mice.

Cardiovascular disease is often associated with premature biological aging and shows evidence of cellular senescence characterized by reduced cell proliferation, growth arrest, apoptosis, elevated DNA damage, and telomere shortening and dysfunction. The miR-34 family is highly conserved at sequence and function levels from nematode to mammals (reviewed in [6]). miR-34 was known to be upregulated during aging and linked with agingassociated pathways, such as DNA damage response, senescence, and cell death (reviewed in [6]).

Other miRNAs are expressionally changed with aging [1] (and reviewed in [6]). It would be important to study the cardiovascular effects of each of these miRNAs. This could result in the identification of multiple therapeutic targets, which could be used in combination to maximize the benefit especially in the elderly.

Boon and colleagues provide an example of a selected miRNA target gene that is therapeutically valuable. Protein phosphatase 1 nuclear-targeting subunit (PNUTS) is a direct miR-34a target and interacts with the telomeric protein telomere repeat factor 2 (TRF2), which is involved in DNA damage repair and telomere maintenance, thereby regulating telomere length [7]. PNUTS directly interacts with phosphatase and tensin homolog (PTEN) and sequesters it in the nucleus, thus reducing PTENdependent apoptosis [8]. Adeno-associ- 


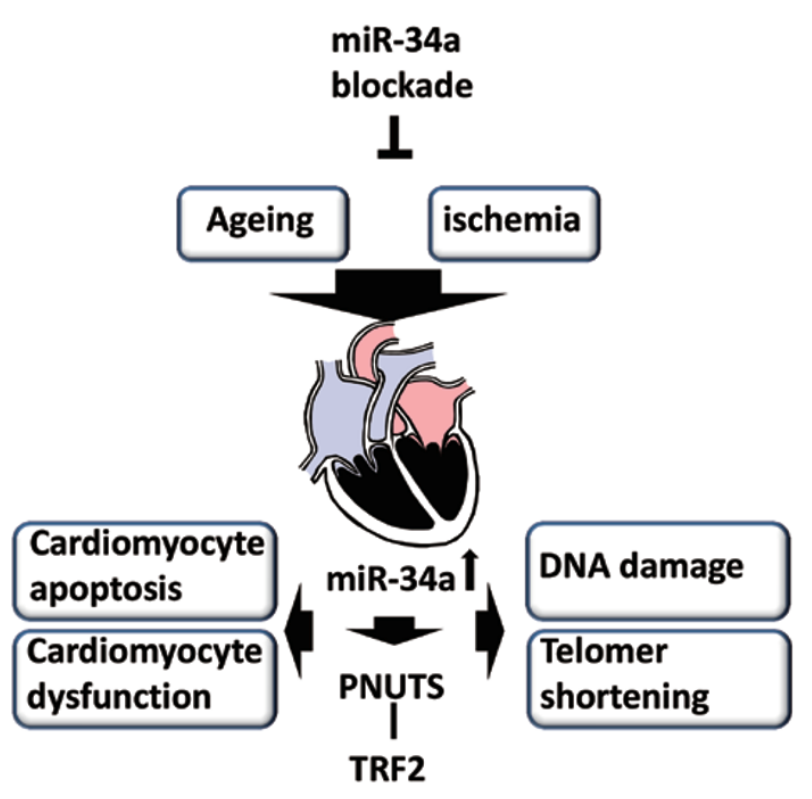

Figure 1 Schematic diagrams of the effects of microRNA-34 (miR-34) inhibition in the aged or ischemic heart. PNUTS, protein phosphatase 1 nuclear-targeting subunit (miR-34 target gene); TRF2, telomeric protein telomere repeat factor 2 .

ated viral delivery of PNUTS improved cardiac function after MI in mice [1].

Cardiac hypertrophy, apoptosis and fibrosis are not exclusive features of aging and more generally present in the "stressed" heart, including after MI and in response to high arterial blood pressure and activated renin-angiotensin system (which are mimicked by the TAC model). However, the authors provide a more specific although indirect evidence of the link between miR-34a and cardiomyocyte aging by looking at the telomere in vitro. Different from other cells, cardiomyocyte cultures can resist for a few days and do not allow mimic senescence by multiple passaging. It would be important to next clarify in vivo whether telomere length is maintained by miR-34a inhibition in aging mice.

Do aging and/or DNA damage and apoptosis-linked pathways induce miR34a expression or does miR34-a causes aging and apoptosis? In fact, it would have been important to extensively investigate the mechanisms regulating miR-34a transcription and maturation in order to understand what is underpinning the observed upregulation of mature miR-34a during cardiac aging and stresses. We speculate that the reported positive feedback loop linking p53, miR-34a and SIRT1 plays a role. In fact, p53 can bind to the miR-34 promoter. In turn, miR-34 recapitulates p53 biological effects inhibiting pro-proliferation and anti-apoptotic genes, including BCL2, and silence information regulator 1 (SIRT1) [1, 9], which limits longevity and promotes post-ischemic angiogenesis and cardiac protection [10, 11]. SIRT1 deacetylates and stabilizes $\mathrm{p} 53$, thus eventually controlling miR34a transcription.

In contrast with the negative cardiac effect observed in mice [1], miRNA-34 ensures long-term maintenance of the brain in Drosophila, where the miR-34 target Eip74EF (an ETS domain transcription factor that is a component of steroid hormone signaling pathways) has been shown to be involved in this protective effect [12]. Hence, it will be important to study the impact of the miRNA-34 family in the mammal brain as well as in other organs.

\section{Costanza Emanueli ${ }^{1,2}$,} Thomas Thum ${ }^{3}$

${ }^{1}$ Laboratory of Vascular Pathology and Regeneration, School of Clinical Sciences, University of Bristol, Bristol, England, BS2 8HW; ${ }^{2}$ Vascular Science, National Heart \& Lung Institute, Imperial College of London, London, England; ${ }^{3}$ Institute of Molecular and Translational Therapeutic Strategies, Hannover Medical School, Hannover, Germany

Correspondence: Costanza Emanueli

Tel: +44 (0)117 3423512; Fax: +44 (0)117 3423904

E-mail: costanza.emanueli@bristol.ac.uk

\section{References}

1 Boon RA, Iekushi K, Lechner S, et al. Nature 2013; 495:107-110.

2 Gullo C, Au M, Feng G, et al. Biochim Biophys Acta 2006; 1765:223-234.

3 Jazbutyte V, Fiedler J, Kneitz S, et al. Age (Dordr) 2013; 35:747-762.

4 Iekushi K, Seeger F, Assmus B, et al. Circulation 2012; 125:1765-1773, S1-S7.

5 Bernardo BC, Gao XM, Winbanks CE, et al. Proc Natl Acad Sci USA 2012; 109:1761517620.

6 Inukai S, Slack F. J Mol Biol 2013 Jan 23. doi:10.1016/j.jmb.2013.01.023

7 Kim H, Lee OH, Xin H, et al. Nat Struct Mol Biol 2009; 16:372-379.

8 Kavela S, Shinde SR, Ratheesh R, et al. Cancer Res 2013; 73:205-214.

9 Raver-Shapira N, Marciano E, Meiri E, et al. Mol Cell 2007; 26:731-743.

10 Potente M, Ghaeni L, Baldessari D, et al. Genes Dev 2007; 21:2644-2658.

11 Hsu CP, Zhai P, Yamamoto T, et al. Circulation 2010; 122:2170-2182.

12 Liu N, Landreh M, Cao K, et al. Nature 2012; 482:519-523. 